\title{
A New Input Constrained Quadratic Tracker for an Unknown Sampled-Data System with an Input to Output Direct Transmission Term
}

\author{
By C. $-Y . W u^{*}$ \\ J. S.-H. Tsai \\ L.-C. Chen \\ S.-M. Guo ${ }^{+}$ \\ L. - S. Shieh \\ T.-J. Tsai
}

\begin{abstract}
A new quadratic digital tracker for efficient tracking control of an unknown sampled-data system with a direct transmission term from an input to output and subject to input constraints is proposed in this paper. First, the observer/Kalman filter identification (OKID) method is utilized to identify an appropriate (low-) order state-space innovation model with a feed-through term, equivalent to the unknown linear system; this identified model is used for the design of the controller and observer. The newly proposed inputconstrained quadratic digital tracker also comprises a new systematic mechanism for tuning the weighting matrix in the cost function of interest. Further, the realizable current output-based digital observer with a direct transmission term is developed for the system whose states are immeasurable. An illustrative example is given to demonstrate the effectiveness of the proposed approach.
\end{abstract}

Keywords: Direct transmission term, Input-constrained quadratic tracker, Observer/Kalman filter identification

\section{Introduction}

The identification of linear/nonlinear systems has been well-studied in the literature (Ljung and Soderstrom, 1983; Goodwin and Sin, 1984; Sinha and Rao, 1991). There have been many successful methods to identify the parameters of a state-space model. Early studies on this subject go back to the work of Ho and Kalman (1965), who considered the concept of minimal

* National Cheng Kung University, Taiwan.

${ }^{\dagger}$ Professor, National Cheng Kung University, Taiwan.

$¥$ National Cheng Kung University, Taiwan.

${ }^{+}$National Cheng Kung University, Taiwan.

${ }^{\circ}$ University of Houston, USA.

- Tung-Fang Design Institute, Taiwan. 
realization of the identified state-space model, i.e. to obtain a model that is both controllable and observable. In this paper, we utilize the observer/Kalman filter identification (OKID) method (Juang, 1994) for system identification. This method is a time-domain technique to identify a discrete input-output mapping in general coordinates, from the input-output sampled data; also, through an extension of the eigensystem realization algorithm (ERA), the method can solve the order-determination problem that arises in the system identification. It has been reported (Juang, 1994) that, by means of the OKID method, a nonlinear system can be identified and represented as a linear model for the design of a controller and an observer.

In order to reduce the effect of the modeling error in the identified model, a sub-optimal digital redesign approach is proposed in this paper, for the design of a digital high-gain observer which improves the digital observer obtained via the OKID method (Juang, 1994). But the current measured output-based observer for estimating the current state in order to determine the current control input requires having the current measured output and the current control input first. Therefore, there is a causal problem for the current outputbased state estimate tracker. An approach will be proposed in this paper to overcome the above causal problem.

As it is well-known, a high-gain tracker (Tsai et al., 2012) can make the system track the desired input rapidly. Nevertheless, due to its high gain, the magnitude of the control input might exceed the physical limits of the actuator input, leading to deterioration of the closed-loop performance. Recently, many researchers have used linear matrix inequality (LMI)-based optimization to design an anti-windup controller for the input-constrained linear systems (Hu et al., 2008; Shieh et al., 1992; Wu and Lu, 2004; Zaccarian and Teel, 2004). In this paper we propose the use of the model identified by the OKID method, to develop an effective optimal linear quadratic digital tracker and its associated observer, for an unknown system with a feed-through term and subject to input constraints. First, we construct a linear quadratic continuous-time performance index (cost function) containing the hard constraints of the actuator inputs (Tarbouriech and Turner, 2009). Since for the digital control of the sampleddata system, a zero-order hold (Z.O.H.) will be placed between the digital controller and the actuator, the input signal to the latter will be a piecewiseconstant signal. As a consequence, the original continuous-time state-space model can be represented by an exactly equivalent discrete-time state-space model.

Then, a discrete-time performance index (cost function) with a constrained input is pre-specified; in addition, a new systematic mechanism is developed for tuning the weighting matrix in the cost function. When the states and model of the sampled-data system with a direct transmission term are not available for measurement, a realizable digital observer is proposed in this paper for the given system.

At last, the digital design method is utilized to develop the inputconstrained observer-based digital controller for the unknown model with a direct transmission term from input to output. This digital controller is capable 
of closely matching the states between the digitally controlled sampled-data model and the theoretically controlled fictitious continuous-time model (Guo et al., 2000). As a result, the proposed input-constrained observer-based linear quadratic digital tracker makes the unknown model show a good performance in tracking the reference signal, while also maintaining the control input within the upper and lower bounds of saturation. To the authors' knowledge, the optimal digital tracker for an unknown model containing a direct transmission term from input to output and subject to input constraints has not been proposed in literature.

This paper is organized as follows. The problem description is given in the next section. In the "Observer/Kalman Filter Identification" section, the OKID method is introduced to determine the discrete-time linear observer with an appropriate (low-) order for an unknown linear system. The following section proposes the design methodology of the discrete-time quadratic tracker for a sampled-data linear model with a direct transmission term from the input to output and subject to input constraints. Section "A Realizable Current-OutBased Digital Observer for the Model with a Feed-Through Term" presents the input-constrained observer-based digital tracker, according to the predictionbased digital observer design, when the system states are immeasurable. Then, the design procedure is summarized and an illustrative example is provided right after. Finally, the conclusions are given at the end.

\section{Problem Description}

Consider the unknown linear system with constrained actuator described by

$$
\begin{gathered}
\dot{x}_{d}(t)=A x_{d}(t)+B u_{d}(t), \quad x_{d}(0)=x_{0}, \\
y_{d}(t)=C x_{d}(t)+D u_{d}(t),
\end{gathered}
$$

where $x_{0}$ is the vector of the initial system states, $u_{d}(t) \in \mathfrak{R}^{m}$ is a piecewiseconstant input vector, such that $u_{d}(t)=u_{d}(k T)$ for $k T \leq t \leq(k+1) T$, and $T>0$ is the sampling period.

First, we use the off-line observer/Kalman filter identification (OKID) method to determine an appropriate (low-) order discrete-time linear observer for the unknown system (1). The identified state space model is formulated as an equivalent linear model with a direct transmission term, which is used to design the input-constrained high-gain digital controller, and the predictionbased digital observer. When the digital control input $u_{d}(k T)$ exceeds the limit of input saturation, $u_{d}(k T)$ will be iteratively adjusted through a proposed algorithm until it is within its specified bounds and minimizes the absolute value of the difference between the control input and the saturation limit as possible. It is desired to propose a new input-constraint current output-based state-estimate tracker for the unknown system with an input to measured output feed-through term. Figure 1 shows the flowchart of the proposed methodology 
for the design of the input-constrained digital tracker and the corresponding prediction-based digital observer.

Figure 1. Observer-Based Input-Constrained Digital Tracker

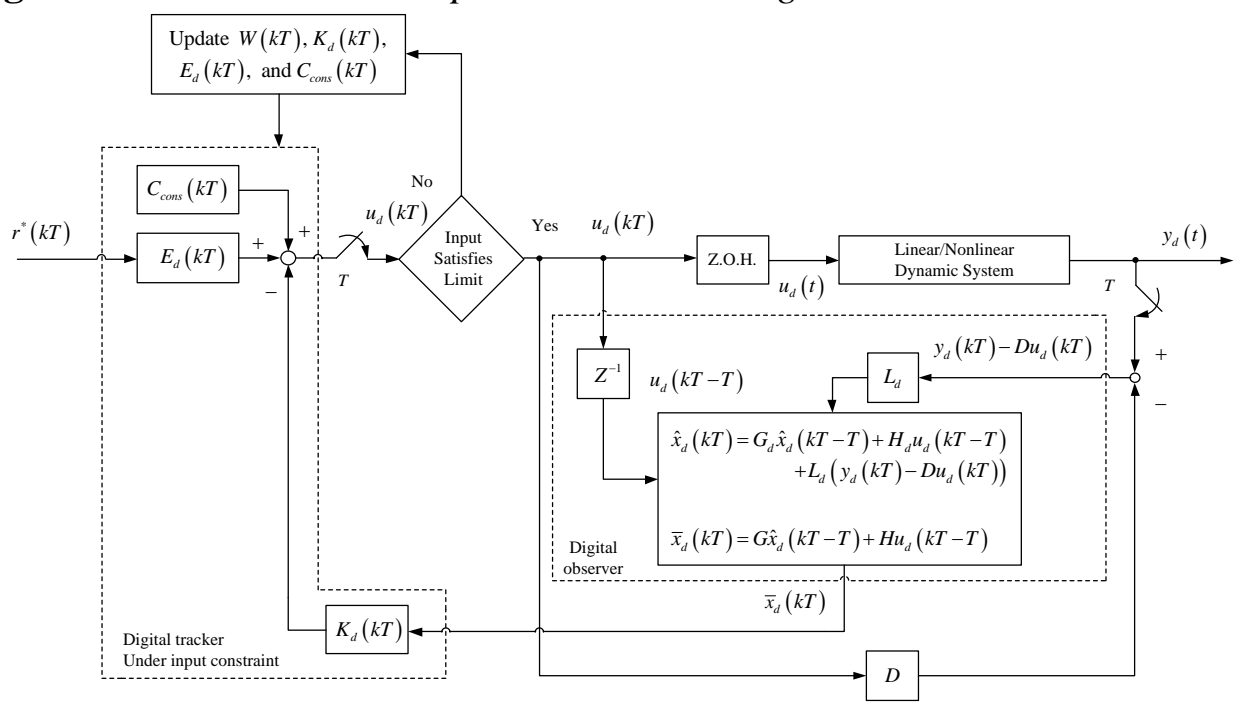

The proposed discrete-time state-feedback control law $u_{d}(t)$ is given by the form

$$
u_{d}(k T)=-K_{d}(k T) x_{d}(k T)+E_{d}(k T) r^{*}(k T)+C_{c o n s}(k T),
$$

where $K_{d}(k T), E_{d}(k T), C_{\text {cons }}(k T)$ will be determined as indicated in what follows, and $r^{*}(k T)$ is a digital reference input vector which, for tracking purpose, is specified as $r^{*}(k T)=r(k T+T)$ [Guo, 2000].

\section{Observer/Kalman Filter Identification}

The off-line OKID (Juang, 1994) method is summarized as follows. The discrete-time state-space model of a multivariable linear system can be represented in the following general form

$$
\begin{gathered}
x(k T+T)=G x(k T)+H u(k T), \\
y(k T)=C x(k T)+D u(k T),
\end{gathered}
$$

where $x(k T) \in \mathfrak{R}^{n}, u(k T) \in \mathfrak{R}^{m}$, and $y(k T) \in \mathfrak{R}^{p}$ are state, output, and control input vectors, respectively, and $G \in \mathfrak{R}^{n \times n}, H \in \mathfrak{R}^{n \times m}$, and $C \in \mathfrak{R}^{p \times n}$ are system, input, and output matrices, respectively. The Hankel matrix obtained from the combined Markov parameters is associated with the system and the observer as

$$
\tilde{H}(k-1)=\left[\begin{array}{cccc}
\Upsilon_{k} & \Upsilon_{k+1} & \cdots & \Upsilon_{k+\beta-1} \\
\Upsilon_{k+1} & \Upsilon_{k+2} & \cdots & \Upsilon_{k+\beta} \\
\vdots & \vdots & \ddots & \vdots \\
\Upsilon_{k+\alpha-1} & \Upsilon_{k+\alpha} & \cdots & \Upsilon_{k+\alpha+\beta-2}
\end{array}\right],
$$


where $\alpha$ and $\beta$ are two sufficiently large but otherwise arbitrary integers, and $\Upsilon_{k}=\left[\begin{array}{lll}C G^{k-1} H & C G^{k-1} F_{o k}\end{array}\right]$, and $F_{o k}$ is the observer gain to be determined based on input and output measurements. When the combined Markov parameters are determined, the Eigen system realization algorithm (ERA) is used to obtain the desired appropriate (low order) $n_{\min }$ and the discrete system and observer realization $\left[G_{o k}, H_{o k}, C_{o k}, D_{o k}, F_{o k}\right]$ through the singular value decomposition (SVD) of the Hankel matrix.

The ERA processes a factorization of the block data matrix in (5), starting from $k=1$, using the singular value decomposition formula $\tilde{H}(0)=V \sum S^{\mathrm{T}}$, where the columns of matrices $V$ and $S$ are orthonormal and $\sum$ is a rectangular matrix of the form

$$
\Sigma=\left[\begin{array}{cc}
\Sigma_{n} & 0 \\
0 & 0
\end{array}\right],
$$

where $\Sigma_{n}=\operatorname{diag}\left[\sigma_{1}, \sigma_{2}, \cdots, \sigma_{n_{\min }}, \sigma_{n_{\min }+1}, \cdots, \sigma_{n}\right]$ contains monotonically non-increasing entries $\sigma_{1} \geq \sigma_{2} \geq \cdots \geq \sigma_{n_{\min }}>>\sigma_{n_{\min }+1} \geq \cdots \geq \sigma_{n}>0$. In order to construct a minimal order observer for the system, let's define $\Sigma_{n_{\min }}=$ $\operatorname{diag}\left[\sigma_{1}, \sigma_{2}, \cdots, \sigma_{n_{\min }}\right]$. The observer with realizations of the system parameters and observer parameters by the ERA is given as

$$
\begin{gathered}
\hat{x}(k T)=G_{o k} \hat{x}(k T-T)+H_{o k} u(k T-T)-F_{o k}[y(k T-T)-\hat{y}(k T-T)], \\
\hat{y}(k T)=C_{o k} \hat{x}(k T)+D_{o k} u(k T),
\end{gathered}
$$

where

$$
\begin{gathered}
G_{o k}=\Sigma_{n_{\min }}^{-1 / 2} V_{n_{\min }}^{\mathrm{T}} \tilde{H}(1) S_{n_{\min }} \Sigma_{n_{\min }}^{-1 / 2}, \\
{\left[H_{o k}, F_{o k}\right]=\text { First }(m+p) \text { columns of } \sum_{n_{\min }}^{1 / 2} S_{n_{\min }}^{\mathrm{T}},} \\
C_{o k}=\text { First } p \text { rows of } V_{n_{\min }} \Sigma_{n_{\min }}^{1 / 2} \text {, and } D_{o k}=\bar{Y}_{0} .
\end{gathered}
$$

In system identification, the SVD is very useful in determining the system order. If the data length is sufficiently long and the order of the observer is sufficiently large, the truncation error is negligible (Juang, 1994).

\section{A New Tracker for the Mathematical Model with a Feed-Through Term}

Through the OKID method (previous section), a discrete-time state-space model (3) equivalent to the unknown system can be identified. Consider the discrete-time state-space model (3), with the input-output feed-through term $D$. The discrete linear quadratic tracker (DLQT) is given by the optimal statefeedback control law

$$
u_{d}(k T)=-K_{d} x_{d}(k T)+E_{d} r^{*}(k T)
$$

with $K_{d} \in \mathfrak{R}^{m \times n}$ the digital feedback gain and $E_{d} \in \mathfrak{R}^{n \times m}$ the forward gain, that minimizes the performance index 


$$
\begin{gathered}
J=\frac{1}{2} \sum_{k=1}^{\infty}\left\{\left[y_{d}(k T)-r(k T)\right]^{\mathrm{T}} Q\left[y_{d}(k T)-r(k T)\right]\right. \\
\left.+u_{d}^{\mathrm{T}}(k T) R u_{d}(k T)\right\} .
\end{gathered}
$$

In the past, the Lagrange multiplier approach and the well-developed optimal control theory (Ogata, 1987) have been used to minimize (9) and determine the optimal quadratic tracker (Tsai et al., 2012) which, due to its high gain (Tsai et al., 2012), often requires high magnitude control inputs to achieve a good tracking performance.

Hence, for the practical implementation of this high gain optimal tracker using a gain-limited actuator, some constraints must be defined as discussed in the following. Let us assume that the system of interest has $m$ control inputs defined as $u_{d}(k T)=\left[u_{1}(k T), u_{2}(k T), \cdots, u_{m}(k T)\right]^{\mathrm{T}}$, and constant upper bound $\left[u_{1}^{\max }, u_{2}^{\max }, \cdots, u_{m}^{\max }\right]$ and lower bound $\left[u_{1}^{\min }, u_{2}^{\min }, \cdots, u_{m}^{\min }\right]$ are independently defined for each input. In other words, the constraints

$$
\left\{\begin{array}{c}
u_{1}^{\min } \leq u_{1}(k T) \leq u_{1}^{\max } \\
u_{2}^{\min } \leq u_{2}(k T) \leq u_{2}^{\max }, \\
\vdots \\
u_{m}^{\min } \leq u_{m}(k T) \leq u_{m}^{\max }
\end{array}\right.
$$

must be satisfied, which can be written as the vector inequality

$$
\underline{u}_{m \times 1} \leq u_{c} \leq \bar{u}_{m \times 1},
$$

where $\underline{u}_{m \times 1}$ and $\bar{u}_{m \times 1}$ are the collection of the lower and upper bounds of the control input, respectively. The constraint (11) should be decomposed into two parts given as

$$
u_{d}-\bar{u} \leq 0
$$

and

$$
-u_{d}+\underline{u} \leq 0
$$

which can be expressed in matrix form as

$$
M u_{d}-N \leq 0
$$

where $M=\left[\begin{array}{c}I_{m \times m} \\ -I_{m \times m}\end{array}\right]$ and $N=\left[\begin{array}{c}\bar{u} \\ -\underline{u}\end{array}\right]$. The inequality constraints $M u_{d}(k T) \leq N$ as in (13) may comprise active constraints and inactive constraints. An inequality $M_{i} u_{d}(k T) \leq N_{i}$ is said to be active if $M_{i} u_{d}(k T)=N_{i}$ and inactive if $M_{i} u_{d}(k T)<N_{i}$, where $M_{i}$ together with $N_{i}$ form the $i^{\text {th }}$ inequality constraint and are the $i^{\text {th }}$ row of $M_{i}$ matrix and the $i^{\text {th }}$ element of $N_{i}$ vector, respectively (Wang, 2009).

Then, considering the constraint (13) and the discrete-time state-space model (3) with a feed-through term, the cost function (9) can be modified as

$$
J=\frac{1}{2} \sum_{k=1}^{k_{f}}\left\{\left[y_{d}(k T)-r(k T)\right]^{\mathrm{T}} Q\left[y_{d}(k T)-r(k T)\right]+u_{d}^{\mathrm{T}}(k T) R u_{d}(k T)\right.
$$




$$
\begin{gathered}
+\left[M_{a c t}(k T) u_{d}(k T)-N_{a c t}(k T)\right]^{\mathrm{T}} W_{a c t}(k T) \\
\left.\times\left[M_{a c t}(k T) u_{d}(k T)-N_{a c t}(k T)\right]\right\},
\end{gathered}
$$

where

$$
M_{i}(k T) u_{d}(k T)-N_{i}(k T)>0 \text { and } w_{i}(k T)>0
$$

for $i \in S_{a c t}(k T), 1 \leq i \leq 2 m$,

$$
M_{i}(k T) u_{d}(k T)-N_{i}(k T) \leq 0 \text { and } w_{i}(k T)=0
$$

for $i \notin S_{a c t}(k T), 1 \leq i \leq 2 m$,

$W=\operatorname{diag}\left[w_{1}, w_{2}, \cdots, w_{m}: w_{m+1}, w_{m+2}, \cdots, w_{2 m}\right] \geq 0$ is a weighting matrix to penalize the cost of inequality constraint, $Q \geq 0$ is the positive semi-definite matrix, $R>0$ is the positive definite matrix, $r(k T) \in \mathfrak{R}^{P}$ is the reference input; $M_{a c t}(k T), W_{a c t}(k T)$, and $N_{a c t}(k T)$ are the corresponding dimension-reduced matrices/vectors of $M, W$, and $N$, which contain only those corresponding terms for $w_{i}>0, i \in S_{a c t}(k T)$. The time interval over which we are interested in the behavior of the plant is $\left[0, k_{f}\right]$, where $k_{f}$ is the final time index.

The active set (Wang, 2009) is to define at each step of an algorithm a set of constraints that is to be treated as the active set. The working set is selected to be a subset of the constraints that are actually active at the current status, and thus the current status is feasible for the working set. While moving on the working set, a new constraint boundary is often encountered. It is necessary to add this constraint to the working set and then proceed to the re-defined working surface.

Now, we have the state equation (3a) and the co-state equation (Lewis and Syrmos, 1995)

$$
\lambda(k T)=G^{\mathrm{T}} \lambda(k T+T)+C^{\mathrm{T}} Q\left[C x_{d}(k T)+D u_{d}(k T)-r(k T)\right],
$$

where $\lambda(k T)$ is the Lagrange multiplier with the stationary condition

$$
\begin{aligned}
& D^{\mathrm{T}} Q C x_{d}(k T)+\left(D^{\mathrm{T}} Q D+R+M_{a c t}^{\mathrm{T}}(k T) W_{a c t}(k T) M_{a c t}(k T)\right) u_{d}(k T) \\
& -D^{\mathrm{T}} Q r(k T)-M_{a c t}^{\mathrm{T}}(k T) W_{a c t}(k T) N_{a c t}(k T)+H^{\mathrm{T}} \lambda(k T+T)=0
\end{aligned}
$$

or

$$
\begin{aligned}
u_{d}(k T) & =-\left(D^{\mathrm{T}} Q D+R+M_{a c t}^{\mathrm{T}}(k T) W_{a c t}(k T) M_{a c t}(k T)\right)^{-1} \\
& \times\left[D^{T} Q\left(C x_{d}(k T)-r(k T)\right)-M_{a c t}^{\mathrm{T}}(k T) W_{a c t}(k T) N_{a c t}(k T)\right. \\
& \left.+H^{\mathrm{T}} \lambda(k T+T)\right]
\end{aligned}
$$

Let $\lambda(k T)$ be denoted as the following form

$$
\lambda(k T)=P(k T) x_{d}(k T)-V(k T),
$$

where $P(k T)$ and $V(k T)$ are parameters to be determined in the sequel. Equation (17) can be shown as

$$
\lambda(k T+T)=P(k T+T) x_{d}(k T+T)-V(k T+T) .
$$


Substituting (18) into (16) yields

$$
\begin{aligned}
u_{d}(k T)=- & K_{d}(k T) x_{d}(k T)-\bar{R}^{-1}(k T) D^{\mathrm{T}} Q r(k T)-\bar{R}^{-1}(k T) H^{\mathrm{T}} \\
& \times V(k T+T)-\bar{R}^{-1} M_{a c t}^{\mathrm{T}}(k T) W_{a c t}(k T) M_{a c t}(k T),
\end{aligned}
$$

where $K_{d}(k T)=\bar{R}^{-1}(k T)\left[\bar{N}^{\mathrm{T}}+H^{\mathrm{T}} P(k T+T) G\right], \bar{R}(k T)=D^{\mathrm{T}} Q D+R$ $+M_{a c t}^{\mathrm{T}}(k T) M_{a c t}(k T) W_{a c t}(k T)$ and $\bar{N}=C^{\mathrm{T}} Q D$.

Equation (15) can be rewritten as

$$
\lambda(k T)=G^{\mathrm{T}} \lambda(k T+T)+C^{\mathrm{T}} Q C x_{d}(k T)+C^{\mathrm{T}} Q D u_{d}(k T)-C^{\mathrm{T}} Q r(k T) .
$$

Similarly, substituting (17) into (20) yields

$$
\begin{gathered}
P(k T) x_{d}(k T)-V(k T)=G^{\mathrm{T}} \lambda(k T+T)+C^{\mathrm{T}} Q C x_{d}(k T) \\
+C^{\mathrm{T}} Q D u_{d}(k T)-C^{\mathrm{T}} Q r(k T) .
\end{gathered}
$$

Subsequently, substituting (19) into (21), one has

$$
\begin{gathered}
P(k T) x_{d}(k T)-V(k T)=G^{\mathrm{T}} \lambda(k T+T)+C^{\mathrm{T}} Q C x_{d}(k T)+C^{\mathrm{T}} Q D \\
\times\left\{-K_{d}(k T) x_{d}(k T)-\bar{R}^{-1}(k T) D^{\mathrm{T}} Q r(k T)-\bar{R}^{-1}(k T) H^{\mathrm{T}} V(k T+T)\right. \\
\left.-\bar{R}^{-1}(k T) M_{a c t}^{\mathrm{T}}(k T) W_{a c t}(k T) M_{a c t}(k T)\right\}-C^{\mathrm{T}} Q r(k T) .
\end{gathered}
$$

Organizing (18) and (22) yields

$$
\begin{gathered}
P(k T) x_{d}(k T)-V(k T)=\left[C^{\mathrm{T}} Q C+G^{\mathrm{T}} P(k T+T) G-\left(G^{\mathrm{T}} P(k T+T) H+\bar{N}\right)\right. \\
\left.K_{d}(k T)\right] x_{d}(k T)+\left[K_{d}^{\mathrm{T}}(k T) D^{\mathrm{T}}-C^{\mathrm{T}}\right] Q r(k T)+\left[K_{d}^{\mathrm{T}}(k T) H^{\mathrm{T}}-G^{\mathrm{T}}\right] \\
\times V(k T+T)+K_{d}^{\mathrm{T}}(k T) M_{a c t}^{\mathrm{T}}(k T) W_{a c t}(k T) M_{a c t}(k T)=0 .
\end{gathered}
$$

Accordingly, one obtains

$$
P(k T)=C^{\mathrm{T}} Q C+G^{\mathrm{T}} P(k T+T) G-\left(G^{\mathrm{T}} P(k T+T) H+\bar{N}\right) K_{d}(k T)
$$

and

$$
\begin{gathered}
V(k T)=\left[C-D K_{d}(k T)\right]^{\mathrm{T}} Q r(k T)+\left[G-H K_{d}(k T)\right]^{\mathrm{T}} V(k T+T) \\
-K_{d}^{\mathrm{T}}(k T) M_{a c t}^{\mathrm{T}}(k T) W_{a c t}(k T) M_{a c t}(k T) .
\end{gathered}
$$

From (19), one has

$$
\begin{gathered}
u_{d}(k T)=-K_{d}(k T) x_{d}(k T)-\bar{R}^{-1}(k T) D^{\mathrm{T}} Q r(k T) \\
-\bar{R}^{-1}(k T) H^{\mathrm{T}} V(k T+T)-\bar{R}^{-1}(k T) M_{a c t}^{\mathrm{T}}(k T) W_{a c t}(k T) M_{a c t}(k T),
\end{gathered}
$$

where

$$
K_{d}(k T)=\bar{R}^{-1}(k T)\left[\bar{N}^{\mathrm{T}}+H^{\mathrm{T}} P(k T+T) G\right] .
$$

Based on (26), (25) becomes

$$
\begin{aligned}
V(k T)= & G^{\mathrm{T}} V(k T+T)+C^{\mathrm{T}} Q r(k T)-K_{d}^{\mathrm{T}}(k T)\left(D^{\mathrm{T}} Q r(k T)\right. \\
& \left.+H^{\mathrm{T}} V(k T+T)+M_{a c t}^{\mathrm{T}}(k T) W_{a c t}(k T) M_{a c t}(k T)\right) .
\end{aligned}
$$

In steady state, (27) and (24) can be respectively rewritten as

$$
V(k T)=\left[I-\left(G-H K_{d}(k T)\right)^{\mathrm{T}}\right]^{-1}\left(C-D K_{d}(k T)\right)^{\mathrm{T}} \operatorname{Qr}(k T)
$$




$$
\begin{aligned}
& -\left[I-\left(G-H K_{d}(k T)\right)^{\mathrm{T}}\right]^{-1} K_{d}^{\mathrm{T}}(k T) \\
& \times M_{a c t}^{\mathrm{T}}(k T) W_{a c t}(k T) M_{a c t}(k T)
\end{aligned}
$$

and

$$
P=C^{\mathrm{T}} Q C+G^{\mathrm{T}} P G-\left(G^{\mathrm{T}} P H+C^{\mathrm{T}} Q D\right) K_{d}(k T) .
$$

By solving (29), one can have the positive definite matrix $P$. Finally, the optimal control law is determined from (19) and (28) as

$$
u_{d}(k T)=-K_{d}(k T) x_{d}(k T)+E_{d}(k T) r(k T)+C_{\text {cons }}(k T),
$$

where

$$
\begin{aligned}
K_{d}(k T)= & \left(D^{\mathrm{T}} Q D+R+M_{a c t}^{\mathrm{T}}(k T) W_{a c t}(k T) M_{a c t}(k T)\right)^{-1} \\
\times & {\left[D^{\mathrm{T}} Q C+H^{\mathrm{T}} P(k T) G\right], } \\
E_{d}(k T)= & \left(D^{\mathrm{T}} Q D+R+M_{a c t}^{\mathrm{T}}(k T) W_{a c t}(k T) M_{a c t}(k T)+H^{\mathrm{T}} P(k T) H\right)^{-1} \\
\times & \left\{D^{\mathrm{T}}+H^{\mathrm{T}}\left[I-\left(G-H K_{d}(k T)\right)^{\mathrm{T}}\right]^{-1}\left(C-D K_{d}(k T)\right)^{\mathrm{T}}\right\} Q \\
C_{c o n s}(k T)= & \left(D^{\mathrm{T}} Q D+R+M_{a c t}^{\mathrm{T}}(k T) W_{a c t}(k T) M_{a c t}(k T)+H^{\mathrm{T}} P(k T) H\right)^{-1} \\
& \times\left\{I-H^{\mathrm{T}}\left[I-\left(G-H K_{d}(k T)\right)^{\mathrm{T}}\right]^{-1} K_{d}^{\mathrm{T}}(k T)\right\} \\
& \times M_{a c t}^{\mathrm{T}}(k T) W_{a c t}(k T) N_{a c t}(k T) .
\end{aligned}
$$

Equations (29)-(33) show that first using $\{Q, R\}$ optimization for the input constraint-free case and then using $W_{a c t}$ to respect the input constraints, where the weighting matrices $\{Q, R\}$ have been retuned in the sense of additive modification, i.e. $\left\{Q_{e}, R_{e}\right\}=\left\{Q, R+M_{a c t}^{\mathrm{T}} W_{a c t} M_{a c t}\right\}$ for the direct transmission matrix $D$-free case and $\left\{Q_{e}, R_{e}\right\}=\left\{Q, R+D^{\mathrm{T}} Q D\right.$ $\left.+M_{a c t}^{\mathrm{T}} W_{a c t} M_{a c t}\right\}$ for the case with $D$ matrix. Besides, an extra compensation term $C_{\text {cons }}$ (33) appears in the modified controller for input-constraint case.

If amplitudes of some components of control input exceed the specified constraints at $t=k^{*} T,(29)$-(33) should be modified in terms of $k^{*} T$ as

$$
u_{d}\left(k^{*} T\right)=-K_{d}\left(k^{*} T\right) x_{d}\left(k^{*} T\right)+E_{d}\left(k^{*} T\right) r\left(k^{*} T\right)+C_{\text {cons }}\left(k^{*} T\right),
$$

where

$$
\begin{gathered}
K_{d}\left(k^{*} T\right)=\left[D^{\mathrm{T}} Q D+R+M_{a c t}^{\mathrm{T}}\left(k^{*} T\right) W_{a c t}\left(k^{*} T\right) M_{a c t}\left(k^{*} T\right)\right]^{-1} \\
\times\left[D^{\mathrm{T}} Q C+H^{\mathrm{T}} P\left(k^{*} T\right) G\right] \\
E_{d}\left(k^{*} T\right)=\left\{D^{\mathrm{T}} Q D+R+M_{a c t}^{\mathrm{T}}\left(k^{*} T\right) W_{a c t}\left(k^{*} T\right) M_{a c t}\left(k^{*} T\right)+H^{\mathrm{T}} P\left(k^{*} T\right) H\right\}^{-1} \\
\times\left\{D^{\mathrm{T}}+H^{\mathrm{T}}\left[I-\left(G-H K_{d}\left(k^{*} T\right)\right)^{\mathrm{T}}\right]^{-1}\left(C-D K_{d}\left(k^{*} T\right)\right)^{\mathrm{T}}\right\} Q
\end{gathered}
$$




$$
\begin{gathered}
C_{\text {cons }}\left(k^{*} T\right)=\left\{\left[D^{\mathrm{T}} Q D+R+M_{\text {act }}^{\mathrm{T}}\left(k^{*} T\right) W_{\text {act }}\left(k^{*} T\right) M_{a c t}\left(k^{*} T\right)\right]+H^{\mathrm{T}} P\left(k^{*} T\right) H\right\}^{-1} \\
\times\left\{I-H^{\mathrm{T}}\left[I-\left(G-H K_{d}\left(k^{*} T\right)\right)^{\mathrm{T}}\right]^{-1} K_{d}^{\mathrm{T}}\left(k^{*} T\right)\right\} \\
\times M_{a c t}^{\mathrm{T}}\left(k^{*} T\right) W_{\text {act }}\left(k^{*} T\right) N_{a c t}\left(k^{*} T\right) .
\end{gathered}
$$

As will be shown in (Tsai et al., 2011), a high-gain controller can be obtained by choosing a sufficiently high ratio of $Q$ to $R$ in (14). A high-gain of this input-constrained controller could (i) lead to a high quality performance on trajectory tracking, while maintaining control inputs within their saturation limits, and (ii) reducing the negative effect of system uncertainties, such as nonlinear perturbations, parameter variations, modeling errors and external disturbances. For these reasons, this input-constrained high-gain digital controller is utilized in our approach.

\section{A Realizable Current-Out-Based Digital Observer for the Model with a Feed-Through Term}

The OKID method gives an appropriate observer/Kalman fitter by using the one-step past output $y_{d}(k T-T)$ to estimate the current state $\hat{x}_{d}(k T)$. Since the current output $y_{d}(k T)$ is available, a current output-based observer will be proposed in this section for the system with the direct transmission term from the input to the measured output, associated with a new approach to overcome the control input causal problem. Let us assume that the state $x_{c}(t)$ of the continuous-time model (1) is immeasurable, and let the continuous-time observer be described by

$$
\begin{gathered}
\dot{\hat{x}}_{c}(t)=A \hat{x}_{c}(t)+B u_{c}(t)+L_{c}\left[y_{c}(t)-\hat{y}_{c}(t)\right], \\
\hat{y}_{c}(t)=C \hat{x}_{c}(t)+D u_{c}(t),
\end{gathered}
$$

where $\hat{x}_{c}(t) \in \mathfrak{R}^{n}$ is the estimated state, $\hat{y}_{c}(t)$ is the output estimated by the observer and $L_{c} \in \mathfrak{R}^{n \times p}$ is the observer gain.

Define the continuous-time state estimate error $e_{c}(t)$ and the discrete-time state estimate error $e_{d}(k T)$ respectively as

$$
\begin{gathered}
e_{c}(t) \equiv x_{c}(t)-\hat{x}_{c}(t), \\
e_{d}(k T) \equiv x_{d}(k T)-\hat{x}_{d}(k T) .
\end{gathered}
$$

The aim of digital redesign (Guo et al., 2000) is to make the discrete-time state estimation error closely match the continuous-time state estimation error at each sampling instant, such as

$$
\left.e_{d}(k T) \cong e_{c}(t)\right|_{t=k T} .
$$

The discrete-time model corresponding to the analog model (1) is given as

$$
x_{d}(k T+T)=G x_{d}(k T)+H u_{d}(k T) \text {, }
$$




$$
y_{d}(k T)=C x_{d}(k T)+D u_{d}(k T) .
$$

After a series of math operations (Guo and Peng, 2005; Tsai et al., 2007), one has

$$
\begin{aligned}
& \begin{aligned}
\hat{x}_{d}(k T+T)=G_{d} \hat{x}_{d}(k T)+H_{d} u_{d}(k T) \\
+L_{d}\left[y_{d}(k T+T)-D u_{d}(k T+T)\right],
\end{aligned} \\
& \hat{y}_{d}(k T+T)=C \hat{x}_{d}(k T+T)+D u_{d}(k T+T),
\end{aligned}
$$

where

$$
\begin{gathered}
L_{d}=\left(G-I_{n}\right) A^{-1} L_{c}\left(I_{p}+C\left(G-I_{n}\right) A^{-1} L_{c}\right)^{-1}, \\
G_{d}=\left(I_{n}-L_{d} C\right) G, H_{d}=\left(I_{n}-L_{d} C\right) H, L_{c}=P_{o} C^{\mathrm{T}} R_{o}^{-1},
\end{gathered}
$$

and

$$
A P_{o}+P_{o} A^{\mathrm{T}}-P_{o} C^{\mathrm{T}} R_{o}^{-1} C P_{o}+Q_{o}=0 .
$$

For the realization of the discrete time observer, represented (41) as

$$
\begin{gathered}
\hat{x}_{d}(k T)=G_{d} \hat{x}_{d}(k T-T)+H_{d} u_{d}(k T-T) \\
+L_{d}\left[y_{d}(k T)-D u_{d}(k T)\right], \\
\hat{y}_{d}(k T)=C \hat{x}_{d}(k T)+D u_{d}(k T) .
\end{gathered}
$$

Equation (42) shows to estimate the current state for determining the current control input that requires having the current measured output and the current control input first. Therefore, there is a causal problem for the current control input. So, the control input $u_{d}(k T)$ in (42) is modified as

$$
\begin{array}{r}
u_{d}(k T) \cong-K_{d} \bar{x}_{d}(k T)+E_{d} r(k T+T)+C_{\text {cons }}(k T) \\
=-K_{d}\left[G \hat{x}_{d}(k T-T)+H u_{d}(k T-T)\right] \\
+E_{d} r(k T+T)+C_{\text {cons }}(k T) .
\end{array}
$$

It is noted that for a system with an input-output direct transmission term, the current-output-based digital observer outperforms the traditional OKIDbased observer described in (Wang et al., 2013).

\section{Design Procedure}

For the input-constraint-free case where $W(k T)=0$, a sufficiently high ratio of $Q$ to $R$ (Tsai, 2011) should be chosen for good tracking performance. If the control input $u_{d}(k T)$ determined by the pre-specified weighting matrices $Q$ and $R$ for $W(k T)=0$ satisfies the input constraint, it is the desired control force naturally. However, such a high ratio weighting matrices $Q$ and $R$ could result in a large-magnitude control input, which may exceed the saturation limits. In the case, the weighting matrix $W(k T)$ or the compact form of the activated components of $W(k T), \quad$ dented by $W_{a c t}(k T)=\operatorname{diag}\left[w_{a c t, 1}(k T), w_{a c t, 2}(k T), \cdots, w_{a c t, \bar{m}(k T)}(k T)\right], \quad$ where $\quad 1 \leq \bar{m}(k T)$ 
$\leq m$, must be searched within a reasonable interval at each sampling instant to have a satisfied input constrained digital controller in terms of $K_{d}(k T)$, $E_{d}(k T), C_{c o n s}(k T), r(k T+T)$ and the estimated state $\bar{x}_{d}(k T)$ in (43), where $\left\{K_{d}(k T), E_{d}(k T), C_{\text {cons }}(k T)\right\} \quad$ are a function of $\left\{Q_{e}, R_{e}(k T)\right\}=\{Q, R$ $\left.+M_{a c t}^{\mathrm{T}}(k T) W_{a c t}(k T) M_{a c t}(k T)\right\}$. Whenever, $\quad u_{d, i}(k T)$ violates the input constraint, it means that the ratio $q_{c, i} / r_{c, i}$ for the input-constraint-free case at $t=k T$ is too high already, where $Q=\operatorname{diag}\left[q_{c, 1}, q_{c, 2}, \cdots, q_{c, p}\right]$ and $R=\operatorname{diag}\left[r_{c, 1}, r_{c, 2}, \cdots, r_{c, m}\right]$. Thus, if $q_{c, i}$ is kept invariant, then the corresponding $r_{c, i}(k T)$ should be appropriately increased to reduce the ratio of $q_{c, i} \quad$ to $\quad r_{e, i}(k T), \quad$ where $\quad R_{e}(k T)=\operatorname{diag}\left[r_{e, 1}(k T), r_{e, 2}(k T), \cdots, r_{e, i}(k T), \cdots\right.$ ,$\left.r_{e, m}(k T)\right]$. For simplicity, assume penalties on costs of each violatedconstraint input are equal, which induces to the case where all activated weighting factors $w_{a c t, i}(k T)$ are all equal. Notice that the weighting matrices $\{Q, R\}$ that appeared in cost function (14) have neither the limitation of equal penalties on costs of each input nor the limitation such as $Q=q_{c} I_{p}$ and $R=r_{c} I_{m}$ required in (Tsai et al., 2011). To deal with the case where penalties on costs of each violated-constraint inputs are not equal, some more sophistical methods (Fogel et al., 1966; Falkenauer, 1997) such as evolutionary programming (EP), and genetic algorithm (GA) may be required. However, the procedure might be time-consuming.

Let the control input constraints be given as $u_{s a t, i}=\left[\bar{u}_{d, i}, \underline{u}_{d, i}\right]$ for $i=1, \cdots, m$, where $\bar{u}_{d, i}$ and $\underline{u}_{d, i}$ represent the upper and lower bounds, respectively, for the $i^{\text {th }}$ input, and define

$$
W(k T)=\text { block diag }\left[W_{\max }(k T) W_{\min }(k T)\right],
$$

where

$$
W_{\max }(k T)=\operatorname{diag}\left[w_{\max , 1}(k T) \quad \cdots \quad w_{\max , i}(k T)\right] \in \mathfrak{R}^{m \times m}
$$

and

$$
W_{\text {min }}(k T)=\operatorname{diag}\left[w_{\min , 1}(k T) \quad \cdots \quad w_{\min , i}(k T)\right] \in \mathfrak{R}^{m \times m},
$$

to be used later. Then, the design procedure is given as follows.

Step 1: Perform the off-line OKID method to determine the appropriate order $n$ and the system matrices gain $(G, H, C, D)$. Determine the redesign observer gain $L_{d}$ and compact parameters $\left(G_{d}, H_{d}\right)$ for the current output-based observer, where

$$
\begin{aligned}
& L_{d}=\left(G-I_{n}\right) A^{-1} L_{c}\left[I_{p}+C\left(G-I_{n}\right) A^{-1} L_{c}\right]^{-1}, \\
& G_{d}=\left(I_{n}-L_{d} C\right) G, H_{d}=\left(I_{n}-L_{d} C\right) H, L_{c}=P_{o} C^{\mathrm{T}} R_{o}^{-1}, \\
& A P_{o}+P_{o} A^{\mathrm{T}}-P_{o} C^{\mathrm{T}} R_{o}^{-1} C P_{o}+Q_{o}=0,
\end{aligned}
$$


and initial value $\hat{x}_{d}(0)=C^{\dagger} y_{d}(0)$.

Step 2: Reconstruct the current output-based observer for the pre-specified case $W_{a c t}(k T)=0$,

$$
\hat{x}_{d}(k T)=G_{d} \hat{x}_{d}(k T-T)+H_{d} u_{d}(k T-T)+L_{d}\left[y_{d}(k T)-D u_{d}(k T)\right],
$$

where

$$
\begin{aligned}
& u_{d}(k T) \cong-K_{d} \bar{x}_{d}(k T)+E_{d} r(k T+T)+C_{c o n s}, \\
& \bar{x}_{d}(k T)=G \hat{x}_{d}(k T-T)+H u_{d}(k T-T), \\
& K_{d}=\left(D^{\mathrm{T}} Q D+R+M_{a c t}^{\mathrm{T}} W_{a c t} M_{a c t}\right)^{-1}\left[D^{\mathrm{T}} Q C+H^{\mathrm{T}} P G\right], \\
& E_{d}=\left(\bar{R}+H^{\mathrm{T}} P H\right)^{-1}\left\{D^{\mathrm{T}}+H^{\mathrm{T}}\left[I-\left(G-H K_{d}\right)^{\mathrm{T}}\right]^{-1}\left(C-D K_{d}\right)^{\mathrm{T}}\right\} Q, \\
& C_{c o n s}=\left(\bar{R}+H^{\mathrm{T}} P H\right)^{-1}\left\{I-H^{\mathrm{T}}\left[I-\left(G-H K_{d}\right)^{\mathrm{T}}\right]^{-1} K_{d}^{\mathrm{T}}\right\} M_{a c t}^{\mathrm{T}} W_{a c t} N_{a c t}, \\
& \bar{R}=D^{\mathrm{T}} Q D+R+M_{a c t}^{\mathrm{T}} W_{a c t} M_{a c t} .
\end{aligned}
$$

Step 3: If $u_{d}(k T)$ exceeds the limit of input saturation, go to Step 4; otherwise, go to Step 5, since it is the desired control force naturally.

Step 4: On-line determine the best weighting factors $w_{i}(k T)$ for the inputconstrained case, where each $w_{i}(k T)$ gets tuned at each time instant $k T$ to meet the input constraints. To achieve the above goal, perform it step by step as follows.

(i) When $u_{d, i}(k T \mid j)$ is outside such bounds, $w_{\max , i}(k T \mid j)$ and $w_{\min , i}(k T \mid j)$ are initially set as

$\left\{\begin{array}{lll}w_{\max , i}(k T \mid j)=r_{c, i} & \text { if } u_{d, i}(k T \mid j)>\bar{u}_{d, i} & \text { for } i=1,2, \cdots m \\ w_{\min , i}(k T \mid j)=r_{c, i} & \text { if } u_{d, i}(k T \mid j)<\underline{u}_{d, i} & \text { for } i=1,2, \cdots m\end{array}\right.$

for the iterative index $j=1$. Re-computes $u_{d, i}(k T \mid j)$ based on the above-tuned weight matrices.

(ii) If re-computed $u_{d, i}(k T \mid j)$ is still outside the input saturation limits, the following method is utilized to iteratively adjust $w_{\max , i}(k T \mid j)$ and $w_{\min , i}(k T \mid j)$ :

Compute $\delta_{i}(k T \mid j)$ as

$$
\delta_{i}(k T \mid j)=\left\{\begin{array}{ll}
u_{d, i}(k T \mid j) / \bar{u}_{d, i} & \text { if } u_{d, i}(k T \mid j)>\bar{u}_{d, i} \\
& \text { for } i=1,2, \cdots, m \\
u_{d, i}(k T \mid j) / \underline{u}_{d, i} & \text { if } u_{d, i}(k T \mid j)<\underline{u}_{d, i} \\
& \text { for } i=1,2, \cdots, m
\end{array} .\right.
$$

Larger $\delta_{i}(k T \mid j)$ implies that $w_{i}(k T \mid j)$ must be significantly increased, so that the tuned $u_{d, i}(k T \mid j)$ could be within the upper 
and lower bounds. On the other hand, smaller $\delta_{i}(k T \mid j)$ indicates that $w_{i}(k T \mid j)$ could be significantly decreased to have $u_{d, i}(k T \mid j)$ to meet the limit condition. To speed up the calculation of the new $w_{i}(k T \mid j)$, an exponential weighting function is used to amplify the effect of $\delta_{i}(k T \mid j)$. When $w_{i}(k T \mid j)$ has been updated more than once, $w_{\max , i}(k T \mid j)$ and $w_{\min , i}(k T \mid j)$ can be modified through the exponential weighting function

$$
\left\{\begin{array}{ll}
w_{\max , i}(k T \mid j):=w_{\max , i}(k T \mid j) \times e^{\tau} & \text { if } u_{d, i}(k T \mid j)>\bar{u}_{d, i} \\
& \text { for } i=1,2, \cdots, m \\
w_{\min , i}(k T \mid j):=w_{\min , i}(k T \mid j) \times e^{\tau} & \text { if } u_{d, i}(k T \mid j)<\underline{u}_{d, i} \\
& \text { for } i=1,2, \cdots, m
\end{array} .\right.
$$

As the rule of thumb, we select $\tau=\delta_{i}(k T \mid j)$ to amplify the effect of $\delta_{i}(k T \mid j)$, so that the re-computed $u_{d, i}(k T \mid j)$ could rapidly meet the limit condition.

(iii) If an over-penalization of the constraint occurs, to minimize the absolute value of the difference between the control input and saturation limit as much as possible, change the exponent-based tuning mechanism of $w_{i}(k T \mid j)$ to the fine tuning mechanism explained in the following

$$
w_{i}(k T \mid j+1):=1.05 \times w_{i}(k T \mid j), \quad \text { for } \quad j:=j^{*}-1, j^{*}, j^{*}+1, \ldots, \bar{j} \text {, }
$$
until $u_{d, i}(k T \mid j)$ satisfies the input constraint for some $j$, or the termination index $\bar{j}$ is reached, where $j^{*}$ is the time index which indicates the first time that the re-computed $u_{d, i}\left(k T \mid j^{*}\right)$ is retuned to within the saturation bound.

Step 5: Update the sampling index $k:=k+1$ and go to Step 2.

From the view point of the multi-objective optimization, it is natural to tune the weighting matrices of Objectives $1,2, \cdots$, sequentially. The purpose of the well-tuned $Q$ and $R$ guarantees Objective 1 (i.e. input-constraint-free tracking performance) is satisfied. Then, tune the weighting matrix $W(k T)$ to yield a satisfied Objective 2 for the input constraints. In general, $r(k T)$ for $k=0,1,2, \cdots$ is a smooth sequence except for some isolated points with acute variations, so it is natural to assume that only Objective 1 is concerned by forcing $W(k T)$ be zero initially. Here, we would like to point out that the optimal weighting matrix $W(k T)$ based on the above mechanism is the optimal one so that the violated input is scaled down to the input constraint at each sampling index $k$ with a minimal variation from the input constraint as possible, which induces a better tracking performance for the input-constrained problem. Furthermore, the proposed mechanism works for the case where the 
input constraints $u_{d, i}^{\max }(k T)$ and $u_{d, i}^{\min }(k T)$ are time varying also, if there is a necessary for some situation.

\section{An Illustrative Example}

Consider a non-square multi-input multi-output controllable and observable unstable system in (1), where

$$
\begin{gathered}
A=\left[\begin{array}{rrrr}
-9.0 & 4.0 & 4.5 & -2.0 \\
-3.0 & 0.4 & 0.7 & -6.0 \\
5.0 & 0.3 & 5.0 & 3.0 \\
4.0 & -2.5 & 2.0 & 3.0
\end{array}\right], B=\left[\begin{array}{rrr}
1.0 & 1.5 & 0.0 \\
0.3 & 2.0 & 0.4 \\
0.3 & -0.3 & 0.0 \\
-0.3 & -1.0 & 0.5
\end{array}\right], \\
C=\left[\begin{array}{rrrr}
1 & 0 & 2 & 1 \\
-1 & 1 & 0 & -1
\end{array}\right], D=\left[\begin{array}{rrr}
0 & 0 & 0 \\
0 & -1 & 0
\end{array}\right],
\end{gathered}
$$

$x_{c}(t) \in \mathfrak{R}^{4}, y_{c}(t) \in \mathfrak{R}^{2}$, and the control input $u_{c}(t) \in \mathfrak{R}^{3}$. The initial state is $x_{c}(0)=\left[\begin{array}{llll}-0.25 & -0.5 & 0.25 & -0.3\end{array}\right]^{\mathrm{T}}$ and the sampling time $T=0.01 \mathrm{sec}$., and assume that the system equation is unknown. The desired reference is given as $r(t)=\left[r_{1}(t), r_{2}(t)\right]^{\mathrm{T}}$, where

$$
r_{1}(t)=\left\{\begin{array}{c}
\cos (2 \pi t), \quad 0 \leq t<1 \mathrm{sec} \\
0.5 \times t^{2}(1-t), \quad 1 \leq t<2 \mathrm{sec} \\
1.5 \times \cos (\pi t) \times \sin (1-3 \pi t), \quad 2 \leq t \leq 3 \mathrm{sec}
\end{array}\right.
$$

and

$$
r_{2}(t)=\left\{\begin{array}{c}
1.2 \times \cos (2 \pi t) \times \sin (1-\pi t), \quad 0 \leq t<1 \mathrm{sec} \\
0.5 \times \cos (2 \pi t), \quad 1 \leq t<2 \mathrm{sec} \\
0.2 \times \sin (4 \pi t)-0.5, \quad 2 \leq t \leq 3 \mathrm{sec}
\end{array}\right.
$$

The input constraints are $N=\left[\begin{array}{c}u_{d}^{\max } \\ -u_{d}^{\min }\end{array}\right]$, where $u_{d}^{\max }=\left[\begin{array}{lll}150 & 20 & 50\end{array}\right]^{\mathrm{T}}$ and $u_{d}^{\min }=\left[\begin{array}{lll}-50 & -20 & -30\end{array}\right]^{\mathrm{T}}$. Then, let the system be excited by the white noise signal $u_{e}(k T)=\left[u_{e 1}(k T), u_{e 2}(k T), u_{e 3}(k T)\right]^{\mathrm{T}}$ with zero mean and a standard deviation of 0.1 .

The identified system matrices and the observer gain for the system are obtained as

$$
G_{o k}=\left[\begin{array}{rrrr}
1.268 & -0.096 & -0.196 & 0.037 \\
0.088 & 1.104 & -0.046 & -0.162 \\
0.200 & -0.022 & 0.951 & -0.117 \\
0.029 & 0.165 & 0.116 & 0.679
\end{array}\right], H_{o k}=\left[\begin{array}{rrr}
-0.001 & -0.005 & -0.000 \\
0.008 & -0.005 & 0.003 \\
-0.004 & -0.004 & -0.002 \\
0.003 & -0.003 & 0.001
\end{array}\right],
$$




$$
\begin{aligned}
& C_{o k}=\left[\begin{array}{rrrr}
-0.879 & 1.143 & -0.435 & 0.519 \\
-1.520 & -0.926 & -0.781 & -0.304
\end{array}\right], D_{o k}=\left[\begin{array}{rrr}
0 & 0 & 0 \\
0 & -1 & 0
\end{array}\right] \text {, and } \\
& F_{o k}=\left[\begin{array}{rr}
1.244 & 1.241 \\
-1.170 & 0.884 \\
-0.603 & -0.662 \\
0.456 & -0.391
\end{array}\right],
\end{aligned}
$$

where the Eigen values of $G_{o k}$ are $\sigma\left(G_{o k}\right)=\sigma\left(e^{A T}\right)=\{0.9125,0.9721,1.0406$ $, 1.0769\}$. The performance of the traditional observer (Juang, 1994) is shown in Figure 2.

Figure 2. Comparison between the Actual System Output and Identified Output Based on the Traditional OKID Method (Juang, 1994): (a) Outputs $y_{d 1}(k T)$ and $y_{o k 1}(k T),(b)$ Outputs $y_{d 2}(k T)$ and $y_{o k 2}(k T)$
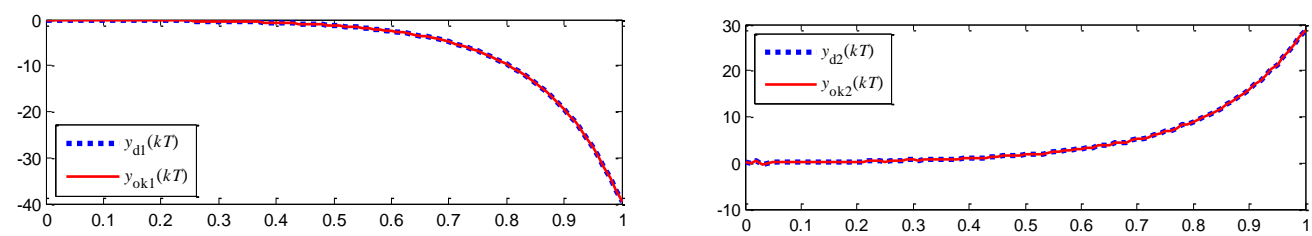

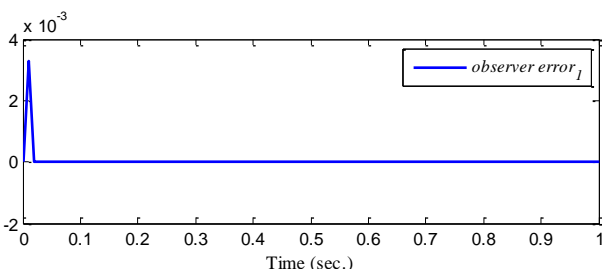

(a)

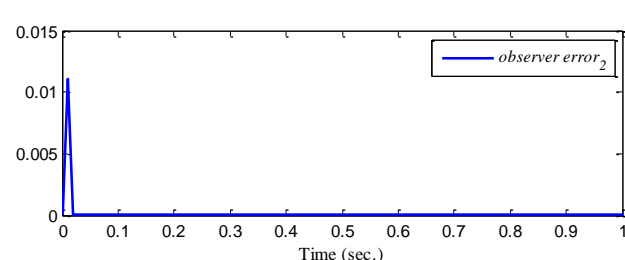

(b)

To further improve the performance of the observer, an improved observer with a high-gain property based on the digital redesign approach has been used, where

$$
\begin{gathered}
G_{d}=\left[\begin{array}{rrrr}
-0.196 & -0.040 & -0.495 & 0.092 \\
-0.062 & 0.007 & -0.068 & -0.270 \\
0.502 & 0.079 & 1.012 & -0.117 \\
0.132 & 0.133 & 0.137 & 0.673
\end{array}\right], \\
H_{d}=10^{-2} \times\left[\begin{array}{rrr}
0.185 & 0.246 & 0.101 \\
-0.040 & 0.128 & 0.010 \\
-0.370 & -0.568 & -0.208 \\
0.232 & -0.364 & 0.028
\end{array}\right] \text {, and } L_{d}=\left[\begin{array}{rr}
-0.352 & -0.497 \\
0.546 & -0.342 \\
0.013 & 0.132 \\
0.039 & 0.028
\end{array}\right],
\end{gathered}
$$

for $Q_{o}=10^{6} I_{4}$ and $R_{o}=I_{2}$. Figure 3 shows the performance of the proposed observer. 
Figure 3. Comparison between the Actual System Output and Identified Output Based on the Proposed Current-Output-Based Observer: (a) Outputs $y_{d 1}(k T)$ and $y_{\text {prop } 1}(k T),(b)$ Outputs $y_{d 2}(k T)$ and $y_{\text {prop } 2}(k T)$
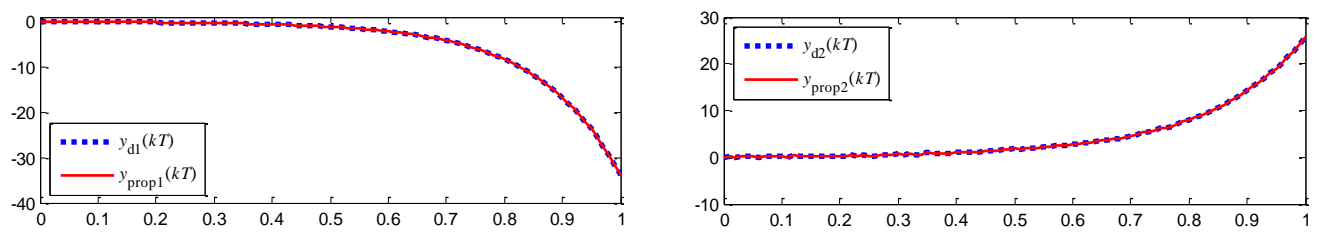

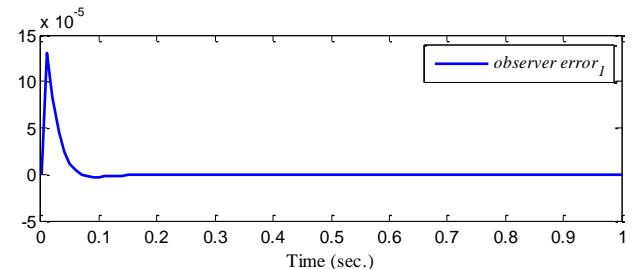

(a)

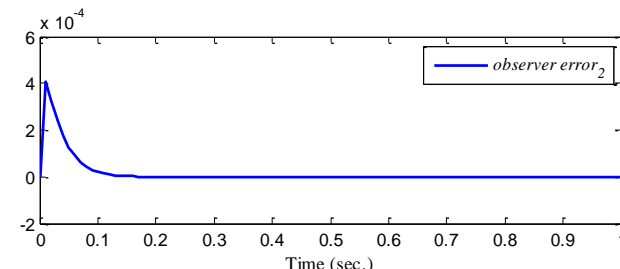

(b)

Figures 2-3 reveal that the proposed observer outperforms the traditional OKID-based observer. Then, according to the "A Realizable Current-OutBased Digital Observer for the Model with a Feed-Through Term" section, the constraint-free feedback gain $K_{d}$ and feed-forward gain $E_{d}$ of the observerbased digital tracker for the system are given as

$$
K_{d}=\left[\begin{array}{rrrr}
-255.771 & 197.072 & 158.857 & -106.324 \\
1.518 & 0.927 & 0.783 & 0.302 \\
451.851 & -232.315 & -453.178 & 308.903
\end{array}\right], E_{d}=\left[\begin{array}{rr}
67.468 & 3.431 \\
0.000 & -1.000 \\
16.889 & -9.032
\end{array}\right] \text {. }
$$

The corresponding computer simulations for the observer-based trackers without and with input constraints are given in Figures 4-5, respectively.

Figure 4. Comparison between Output, Reference, and Control Input without Input Constraint: (a) $y_{d 1}(k T), y_{d 2}(k T), r_{1}(k T)$, and $r_{2}(k T),(b) u_{d 1}(k T)$, $u_{d 2}(k T)$, and $u_{d 3}(k T)$

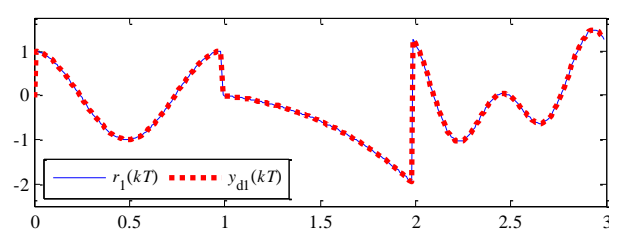

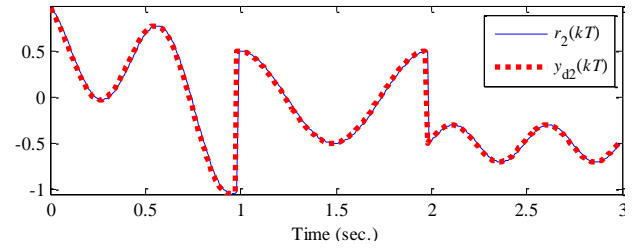

(a)
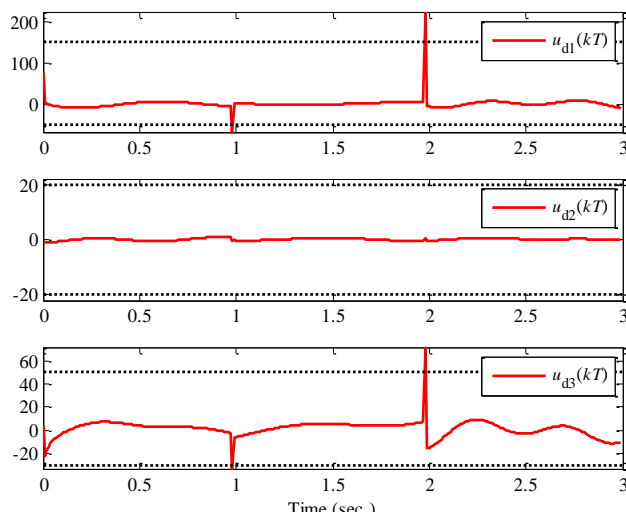

(b) 
Figure 5. Comparison between Output, Reference, and Control Input under Input Constraint: (a) $y_{d 1}(k T), y_{d 2}(k T), r_{1}(k T)$, and $r_{2}(k T),(b) u_{d 1}(k T)$, $u_{d 2}(k T)$, and $u_{d 3}(k T)$

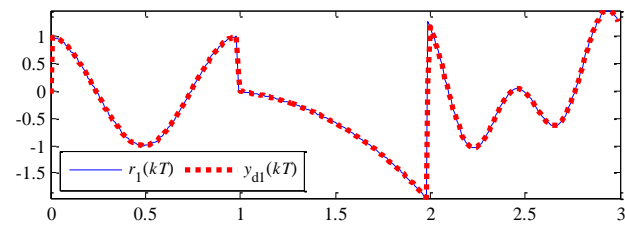

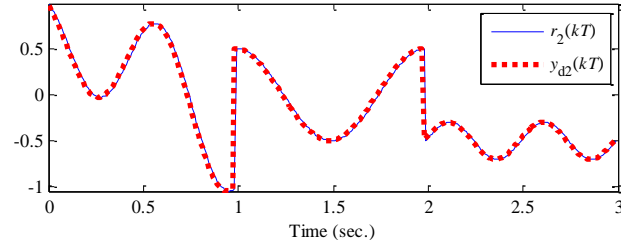

(a)
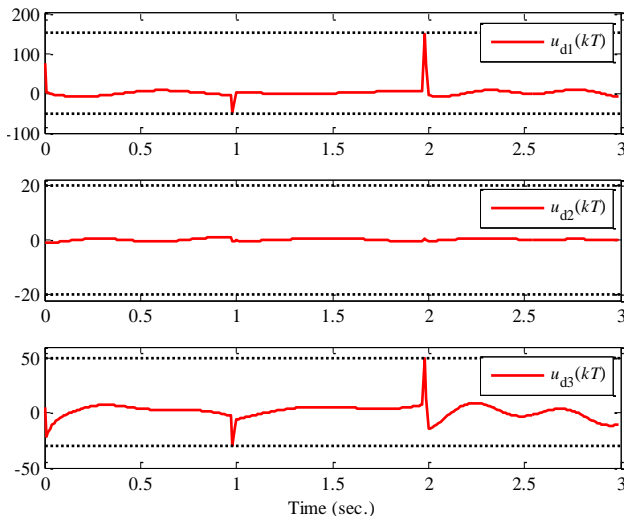

(b)

Figure 5 shows that $u_{d}(k T)=\left[u_{d 1}(k T), u_{d 2}(k T), u_{d 3}(k T)\right]^{T} \quad$ are compressed under the boundary for both channels successfully with a good tracking performance even though the system equation is unknown.

\section{Conclusions}

This paper presents a new input-constrained discrete linear quadratic observer-based tracker for an unknown sampled-data system with a direct transmission term from input to output. The input-output causal problem induced by the current output-based observer associated with the tracker is then overcome by the proposed realization. The newly derived Riccati equation for the input-constraint system with an input-output direct feed-through the term shows the proposed optimal control is optimal in the sense of minimizing the input-constraint-free quadratic cost function in terms of the equivalent weighting matrices $\left\{Q_{e}, R_{e}\right\}$. Although, based on our pre-study, an explicit connection of the equivalent weighting matrix pairs between the QP algorithmbased optimization and the linear quadratic Riccati equation-based optimization for the system with a non-vanished $D$ matrix is supposed to be available, but it still needs a great effort to prove and verify it. Besides, due to our best knowledge, no literature investigates this topic, so it will be considered as a further research topic. Simulation results show that the proposed inputconstrained observer-based linear quadratic digital tracker demonstrates good tracking performance, while maintaining the control input within its lower and upper bounds. 


\section{Acknowledgments}

This work was supported by Ministry of Science and Technology of Republic of China under contacts MOST 103-2221-E-006-023 and NSC 1022221-E-006-208-MY3.

\section{References}

Falkenauer, E. 1997. Genetic Algorithms and Grouping Problems. Chichester, England: John Wiley \& Sons Ltd, New York.

Fogel, L. J., Owens, A. J., and Walsh, M. J. 1996. Artificial Intelligence through Simulated Evolution. John Wiley, New York.

Goodwin, G. C. and Sin, K. S. 1984. Adaptive Filtering Prediction and Control. Prentice Hall, New Jersey.

Guo, S. M. and Peng, Z. H. 2005. An observer-based decentralized tracker for sampled-data systems: an evolutionary programming approach. Int. J. Gen. Syst. 34, (Aug. 2005), 421-449. DOI=10.1080/03081070500132468.

Guo, S. M., Shieh, L. S., Chen, G., and Lin, C. F. 2000. Effective chaotic orbit tracker: A prediction-based digital redesign approach. IEEE Transactions on Circuits and Systems-I, Fundamental Theory and Applications. 47, 11 (Nov. 2000), 15571570. DOI $=10.1109 / 81.895324$.

Ho, B. L. and Kalman, R. E. 1965. Effective construction of linear state-variable models from input-output data. Proceedings of the $3 r d$ Annual Allerton Conference on Circuits and System Theory, Monticello, IL: University of Illinois. 449-459.

Hu, T., Teel, A.R., and Zaccarian, L. 2008. Anti-windup synthesis for linear control systems with input saturation: Achieving regional, nonlinear performance. Automatica. 44, 2 (Feb. 2008), 512-519. DOI= 10.1016/j. automatica.2007.06.003

Juang, J. N. 1994. Applied System Identification. Prentice Hall, New Jersey.

Lewis, F. L. and Syrmos, V. L. 1995. Optimal Control. John Wiley \& Sons, New Jersey.

Ljung, L. and Soderstrom, T. 1983. Theory and Practice of Recursive Identification. MIT press, Cambridge, Mass.

Ogata, K. 1987. Discrete-time Control Systems. Prentice-Hall, Englewood Cliffs, N.J.

Shieh, L. S., Chen, G., and Tsai, J. S. H. 1992. Hybrid suboptimal control of multi-rate multi-loop sampled-data systems. International Journal of Systems Science. 23, 6 (Jun, 1992), 839-854. DOI= 10.1080/00207729208949253.

Sinha, N. K. and Rao, G. P. 1991. Identification of Continuous-time Systems: Methodology and Computer Implementation. Kluwer Academic Publishers, London.

Tarbouriech, S., and Turner, M. 2009. Anti-windup design: An overview of some recent advances and open problems. IET Control Theory and Applications. 3, 1 (Jan. 2009), 1-19. DOI= 10.1049/iet-cta:20070435.

Tsai, J. S. H., Chien, T. H., Guo, S. M., Chang, Y. P., and Shieh, L. S. 2007. Statespace self-tuning control for stochastic chaotic fractional-order chaotic systems. IEEE Transactions on Circuits and Systems Part I: Regular Papers. 54, 3 (Mar. 2007), 632-642. DOI= 10.1109/TCSI.2006.888668. 
Tsai, J. S. H., Du, Y. Y., Zhuang, W. Z., Guo, S. M., Chen, C. W., Shieh, L. S. 2011. Optimal anti-windup digital redesign of multi-input multi-output control systems under input constraints. IET Control Theory Applications. 5, 3 (Feb. 2011), 447464. DOI=10.1049/iet-cta.2010.0020.

Tsai, J. S. H., Chen, C. H., Lin, M. J., Guo, S. M., and Shieh, L. S. 2012. Novel quadratic tracker and observer for the equivalent model of the sampled-data linear singular system. Applied Mathematical Sciences. 6, 68 (Aug. 2012), 3381-3409.

Wang, L. P. 2009. Model Predictive Control System Design and Implementation using MATLAB. Springer, London.

Wang, J. H., Tsai, J. S. H., Huang, J. S., Guo, S. M., and Shieh, L. S. 2013. A loworder active fault-tolerant state space self-tuner for the unknown sampled-data nonlinear singular system using OKID and modified ARMAX model-based system identification. Applied Mathematical Modelling. 37, 3 (Feb. 2013), 12421274. DOI= 10.1016/j.apm.2012.03.035.

$\mathrm{Wu}, \mathrm{F}$. and Lu, B. 2004. Anti-windup control design for exponentially unstable LTI systems with actuator saturation. Systems \& Control Letters. 52, 4 (July 2004), 305-322. DOI= 10.1016/j.sysconle.2004.02.007.

Zaccarian, L. and Teel, A.R. 2004. Nonlinear scheduled anti-windup design for linear systems. IEEE Transactions on Automatic Control. 49, 11 (Nov. 2004), 20552061. DOI= 10.1109/TAC.2004.837539. 